\title{
Analytical Approximation to the Solution of Nonlinear Blasius' Viscous Flow Equation by LTNHPM
}

\author{
Hossein Aminikhah \\ Department of Applied Mathematics, Faculty of Mathematical Sciences, University of Guilan, \\ P.O. Box 1914, Rasht, Iran \\ Correspondence should be addressed to Hossein Aminikhah, hossein.aminikhah@gmail.com \\ Received 16 November 2011; Accepted 8 December 2011 \\ Academic Editor: S. Zhang \\ Copyright (C) 2012 Hossein Aminikhah. This is an open access article distributed under the \\ Creative Commons Attribution License, which permits unrestricted use, distribution, and \\ reproduction in any medium, provided the original work is properly cited.

\begin{abstract}
Laplace transform and new homotopy perturbation methods are adopted to study Blasius' viscous flow equation analytically. The solutions approximated by the proposed method are shown to be precise as compared to the corresponding results obtained by Howarth's numerical method. A high accuracy of the new method is evident.
\end{abstract}

\section{Introduction}

One of the well-known equations arising in fluid mechanics and boundary layer approach is Blasius' differential equation. Blasius [1] in 1908 found the exact solution of boundary layer equation over a flat plate. Afterwards it has been solved by Howarth [2] by means of some numerical methods. The solution of Blasius equation was studied recently by AbuSitta for the mixing layers of fluid past a flat plate and the existence of a solution well established in [3]. Asaithambi [4] presented an effective finite difference method which has improved the previous numerical methods by reducing the amount of computational work. Recently, He [5], Abbasbandy [6], Esmaeilpour and Ganji [7] obtained an approximate solution for Blasius equation using HPM, ADM, and VIM. In this work, we obtain an analytical approximation to the solution of the classical Blasius flat-plate problem using combination of Laplace transform and new homotopy perturbation method (LTNHPM). The results obtained via LTNHPM are compared with the numerical solutions [2] which confirms the validity of the proposed method. 


\section{Governing Equations}

Boundary layer flow over a flat plate is governed by the continuity and the Navier-Stokes equations. For a two-dimensional, steady-state, incompressible flow with zero pressure gradient over a flat plate, governing equations are simplified to

$$
\begin{gathered}
\frac{\partial u}{\partial x}+\frac{\partial v}{\partial y}=0 \\
u \frac{\partial u}{\partial x}+v \frac{\partial v}{\partial y}=v \frac{\partial^{2} u}{\partial y^{2}}
\end{gathered}
$$

The boundary conditions are

$$
y=0, \quad u=v=0, \quad y=\infty, \quad u=U_{\infty} .
$$

Assuming that the leading edge of the plate is $x=0$ and the plate is infinity long, this system can be simplified further to an ordinary differential equation. To do this, we have an equation that reads

$$
\delta \approx \sqrt{\frac{v x}{U_{\infty}}}
$$

To make this quantity dimensionless, it can be divided by $y$ to obtain

$$
\eta=y \sqrt{\frac{U_{\infty}}{v x}}, \quad \varphi=\sqrt{v x U_{\infty}} f(\eta),
$$

where $f(\eta)$ is the dimensionless stream function. The velocity component $u$ which is equal to $\partial \varphi / \partial y$ can be expressed as follows:

$$
u=\frac{\partial \varphi}{\partial y}=\frac{\partial \varphi}{\partial \eta} \frac{\partial \eta}{\partial y}=\sqrt{v x U_{\infty}} f^{\prime}(\eta) \cdot \sqrt{\frac{U_{\infty}}{v x}} .
$$

So $u=U_{\infty} f^{\prime}(\eta)$. Also the transverse velocity component can be expressed as

$$
v=-\frac{\partial \varphi}{\partial x}=\frac{1}{2} \sqrt{\frac{\nu U_{\infty}}{x}}\left(\eta f^{\prime}(\eta)-f(\eta)\right)
$$


Now, inserting (2.5) and (2.6) into the second boundary layer flow equation

$$
-\frac{U_{\infty}^{2}}{2 x} \eta f^{\prime}(\eta) f^{\prime \prime}(\eta)+\frac{U_{\infty}^{2}}{2 x}\left(\eta f^{\prime}(\eta)-f(\eta)\right)=v \frac{U_{\infty}^{2}}{x v} f^{\prime \prime \prime}(\eta)
$$

therefore,

$$
f^{\prime \prime \prime}(\eta)+\frac{1}{2} f(\eta) f^{\prime \prime}(\eta)=0
$$

with boundary equations

$$
\eta=0, \quad f=\frac{d f}{d \eta}=0, \quad \eta \longrightarrow \infty, \quad \frac{d f}{d \eta}=1
$$

In 1908, Blasius [1] provided a solution in the following form:

$$
f(\eta)=\sum_{k=0}^{\infty}\left(-\frac{1}{2}\right) \frac{A_{k} \sigma^{k+1}}{(3 k+2) !} \eta^{3 k+2}
$$

where $\sigma=f^{\prime \prime}(0), A_{0}=A_{1}=1, A_{k}=\sum_{r=0}^{k-1}\left(\begin{array}{c}3 k-1 \\ 3 r\end{array}\right) A_{r} A_{k-r-1}(k \geq 2)$.

Blasius evaluated $\sigma$ by demonstrating another approximation of $f(\eta)$ at large $\eta$. Then, by means of matching two different approximations at a proper point, he obtained the numerical result $\sigma=0.332$. In 1938, by means of a numerical technique, Howarth [2] gained a more accurate value $\sigma=0.332057$ utilized to solve Blasius equation (2.8).

\section{Analysis of the Method}

To illustrate the basic ideas of this method, let us consider the following nonlinear differential equation

$$
A(u)-f(r)=0, \quad r \in \Omega,
$$

with the following initial conditions

$$
u(0)=\alpha_{0}, u^{\prime}(0)=\alpha_{1}, \ldots, u^{(n-1)}(0)=\alpha_{n-1},
$$

where $A$ is a general differential operator and $f(r)$ is a known analytical function. The operator $A$ can be divided into two parts, $L$ and $N$, where $L$ is a linear and $N$ is a nonlinear operator. Therefore, (3.1) can be rewritten as

$$
L(u)+N(u)-f(r)=0 .
$$


By the NHPM [8], we construct a homotopy $U(r, p): \Omega \times[0,1] \rightarrow \mathbb{R}$, which satisfies

$$
H(U, p)=(1-p)\left[L(U)-u_{0}\right]+p[A(U)-f(r)]=0, \quad p \in[0,1], r \in \Omega,
$$

or equivalently,

$$
H(U, p)=L(U)-u_{0}+p u_{0}+p[N(U)-f(r)]=0,
$$

where $p \in[0,1]$ is an embedding parameter and $u_{0}$ is an initial approximation of solution of (3.1). Clearly, we have, from (3.4) and (3.5),

$$
\begin{aligned}
H(U, 0) & =L(U)-u_{0}=0, \\
H(U(x), 1) & =A(U)-f(r)=0 .
\end{aligned}
$$

By applying Laplace transform on both sides of (3.5), we have

$$
\mathcal{L}\left\{L(U)-u_{0}+p u_{0}+p[N(U)-f(r)]\right\}=0 .
$$

Using the differential property of Laplace transform, we have

$$
s^{n} \mathcal{L}\{U\}-s^{n-1} U(0)-s^{n-2} U^{\prime}(0)-\cdots-U^{(n-1)}(0)=\mathcal{L}\left\{u_{0}-p u_{0}+p[N(U)-f(r)]\right\},
$$

or

$$
\mathcal{L}\{U\}=\frac{1}{s^{n}}\left\{s^{n-1} U(0)+s^{n-2} U^{\prime}(0)+\cdots+U^{(n-1)}(0)+\mathcal{L}\left\{u_{0}-p u_{0}+p[N(U)-f(r)]\right\}\right\} .
$$

By applying inverse Laplace transform on both sides of (3.9), we have

$$
U=\mathcal{L}^{-1}\left\{\frac{1}{s^{n}}\left\{s^{n-1} U(0)+s^{n-2} U^{\prime}(0)+\cdots+U^{(n-1)}(0)+\mathcal{L}\left\{u_{0}-p u_{0}+p[N(U)-f(r)]\right\}\right\}\right\} .
$$

According to the HPM, we can first use the embedding parameter $p$ as a small parameter and assume that the solutions of (3.10) can be represented as a power series in $p$ as

$$
U(x)=\sum_{n=0}^{\infty} p^{n} U_{n}
$$


Now, let us write the (3.10) in the following form:

$$
\begin{aligned}
\sum_{n=0}^{\infty} p^{n} U_{n}=\mathcal{L}^{-1}\left\{\frac { 1 } { s ^ { n } } \left\{s^{n-1} U(0)+s^{n-2} U^{\prime}(0)+\cdots+U^{(n-1)}(0)\right.\right. \\
\left.\left.+\mathcal{L}\left\{u_{0}-p u_{0}+p\left[N\left(\sum_{n=0}^{\infty} p^{n} U_{n}\right)-f(r)\right]\right\}\right\}\right\} .
\end{aligned}
$$

Comparing coefficients of terms with identical powers of $p$, leads to

$$
\begin{aligned}
p^{0}: U_{0} & =\mathcal{L}^{-1}\left\{\frac{1}{s^{n}}\left(s^{n-1} U(0)+s^{n-2} U^{\prime}(0)+\cdots+U^{(n-1)}(0)+\mathcal{L}\left\{u_{0}\right\}\right)\right\}, \\
p^{1}: U_{1} & =\mathcal{L}^{-1}\left\{\frac{1}{s^{n}}\left(\mathcal{L}\left\{N\left(U_{0}\right)-u_{0}-f(r)\right\}\right)\right\}, \\
p^{2}: U_{2} & =\mathcal{L}^{-1}\left\{\frac{1}{s^{n}}\left(\mathcal{L}\left\{N\left(U_{0}, U_{1}\right)\right\}\right)\right\}, \\
p^{3}: U_{3} & =\mathcal{L}^{-1}\left\{\frac{1}{s^{n}}\left(\mathcal{L}\left\{N\left(U_{0}, U_{1}, U_{2}\right)\right\}\right)\right\}, \\
& \vdots \\
p^{j}: U_{j} & =\mathcal{L}^{-1}\left\{\frac{1}{s^{n}}\left(\mathcal{L}\left\{N\left(U_{0}, U_{1}, U_{2}, \ldots, U_{j-1}\right)\right\}\right)\right\},
\end{aligned}
$$

Suppose that the initial approximation has the form $U(0)=u_{0}=\alpha_{0}, U^{\prime}(0)=$ $\alpha_{1}, \ldots, U^{(n-1)}(0)=\alpha_{n-1}$; therefore, the exact solution may be obtained as follows:

$$
u=\lim _{p \rightarrow 1} U=U_{0}+U_{1}+U_{2}+\cdots
$$

\section{LTNHPM Applied to the Nonlinear Blasius Ordinary Differential Equation}

Consider the nonlinear Blasius ordinary differential equation (2.8). For solving this equation by applying the new homotopy perturbation method, we construct the following homotopy:

$$
H(F(\eta), p)=F^{\prime \prime \prime}(\eta)-f_{0}(\eta)+p\left(f_{0}(\eta)+\frac{1}{2} F(\eta) F^{\prime \prime}(\eta)\right)=0
$$

where $p \in[0,1]$ is an embedding parameter and $f_{0}(\eta)$ is an initial approximation of solution of (2.8). 


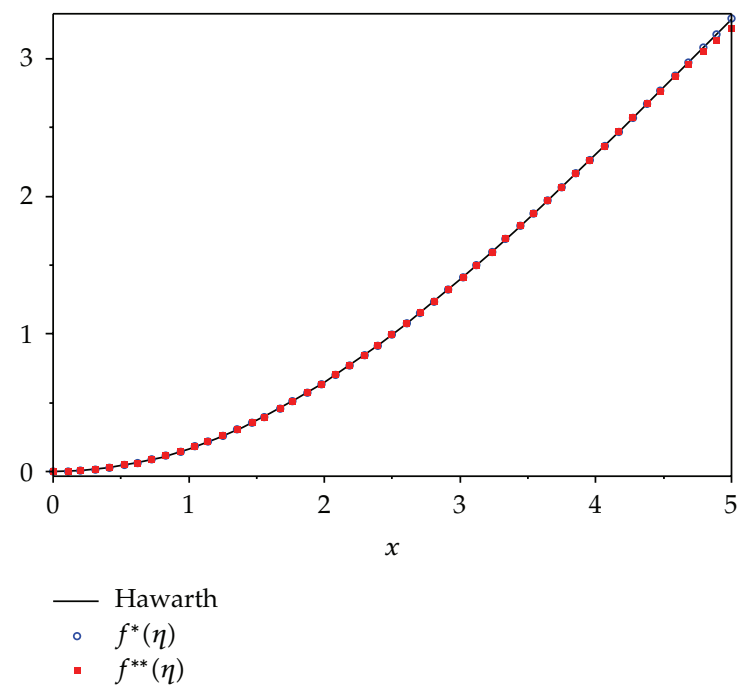

Figure 1: The comparison of answers obtained by LTNHPM and Howarth's results for $f(\eta)$.

Clearly, we have from (4.1)

$$
\begin{aligned}
& H(F(\eta), 0)=F^{\prime \prime \prime}(\eta)-f_{0}(\eta)=0 \\
& H(F(\eta), 1)=F^{\prime \prime \prime}(\eta)+\frac{1}{2} F(\eta) F^{\prime \prime}(\eta)=0 .
\end{aligned}
$$

By applying Laplace transform on both sides of (4.1), we have

$$
\mathcal{L}\left\{F^{\prime \prime \prime}(\eta)-f_{0}(\eta)+p\left(f_{0}(\eta)+\frac{1}{2} F(\eta) F^{\prime \prime}(\eta)\right)\right\}=0 .
$$

Using the differential property of Laplace transform, we have

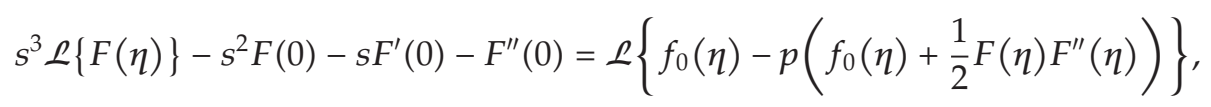

or

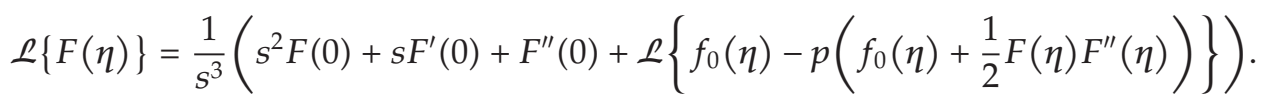

By applying inverse Laplace transform on both sides of (4.5), we have

$$
F(\eta)=\mathcal{L}^{-1}\left\{\frac{1}{s^{3}}\left(s^{2} F(0)+s F^{\prime}(0)+F^{\prime \prime}(0)+\mathcal{L}\left\{f_{0}(\eta)-p\left(f_{0}(\eta)+\frac{1}{2} F(\eta) F^{\prime \prime}(\eta)\right)\right\}\right)\right\}
$$




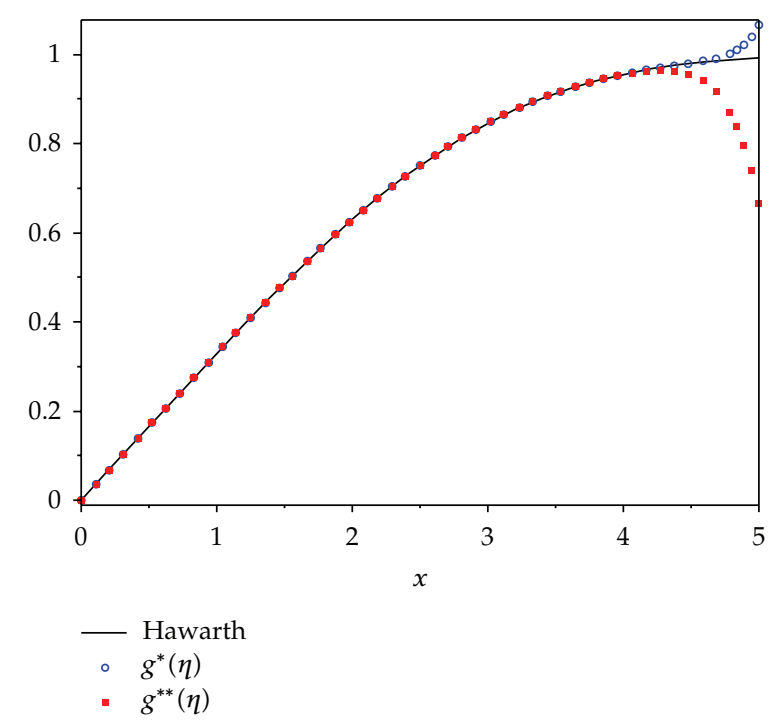

Figure 2: The comparison of answers obtained by LTNHPM and Howarth's results for $f^{\prime}(\eta)$.

According to the HPM, we use the embedding parameter $p$ as a small parameter and assume that the solutions of (4.6) can be represented as a power series in $p$ as

$$
F(\eta)=\sum_{n=0}^{\infty} p^{n} F_{n}(\eta)
$$

Substituting (4.7) into (4.6) and equating the terms with the identical powers of $p$ lead to

$$
\begin{aligned}
p^{0}: F_{0}(\eta) & =\mathcal{L}^{-1}\left\{\frac{F(0)}{s}+\frac{F^{\prime}(0)}{s^{2}}+\frac{F^{\prime \prime}(0)}{s^{3}}+\mathcal{L}\left\{f_{0}(\eta)\right\}\right\}, \\
p^{1}: F_{1}(\eta) & =\mathcal{L}^{-1}\left\{-\frac{1}{s^{3}}\left(\mathcal{L}\left\{f_{0}(\eta)+\frac{1}{2} F_{0}(\eta) F_{0}^{\prime \prime}(\eta)\right\}\right)\right\}, \\
p^{2}: F_{2}(\eta) & =\mathcal{L}^{-1}\left\{-\frac{1}{2 s^{3}}\left(\mathcal{L}\left\{F_{0}(\eta) F_{1}^{\prime \prime}(\eta)+F_{1}(\eta) F_{0}^{\prime \prime}(\eta)\right\}\right)\right\}, \\
p^{3}: F_{3}(\eta) & =\mathcal{L}^{-1}\left\{-\frac{1}{2 s^{3}}\left(\mathcal{L}\left\{F_{0}(\eta) F_{2}^{\prime \prime}(\eta)+F_{1}(\eta) F_{1}^{\prime \prime}(\eta)+F_{2}(\eta) F_{0}^{\prime \prime}(\eta)\right\}\right)\right\}, \\
& \vdots \\
p^{j}: F_{j}(\eta) & =\mathcal{L}^{-1}\left\{-\frac{1}{2 s^{3}}\left(\mathcal{L}\left\{\sum_{k=0}^{j-1} F_{k}(\eta) F_{j-k-1}^{\prime \prime}(\eta)\right\}\right)\right\},
\end{aligned}
$$


Table 1: Comparison between the Howarth and LTNHPM methods for $f(\eta)$.

\begin{tabular}{lccc}
\hline$\eta$ & Howarth [2] & $f^{*}(\eta)$ & $f^{* *}(\eta)$ \\
\hline 0 & 0.00000 & 0.00000 & 0.00000 \\
0.2 & 0.00664 & 0.00664 & 0.00664 \\
0.4 & 0.02656 & 0.02656 & 0.02656 \\
0.6 & 0.05974 & 0.05973 & 0.05973 \\
0.8 & 0.10611 & 0.10611 & 0.10611 \\
1 & 0.16557 & 0.16557 & 0.16557 \\
1.2 & 0.23795 & 0.23795 & 0.23795 \\
1.4 & 0.32298 & 0.32298 & 0.32298 \\
1.6 & 0.42032 & 0.42032 & 0.42032 \\
1.8 & 0.52952 & 0.52952 & 0.52952 \\
2 & 0.65003 & 0.65002 & 0.65002 \\
2.2 & 0.78120 & 0.78119 & 0.78119 \\
2.4 & 0.92230 & 0.92228 & 0.92228 \\
2.6 & 1.07252 & 1.07250 & 1.07250 \\
2.8 & 1.23099 & 1.23098 & 1.23098 \\
3 & 1.39682 & 1.39681 & 1.39681 \\
3.2 & 1.56911 & 1.56909 & 1.56909 \\
3.4 & 1.74696 & 1.74694 & 1.74695 \\
3.6 & 1.92954 & 1.92951 & 1.92952 \\
3.8 & 2.11605 & 2.11596 & 2.11602 \\
4 & 2.30576 & 2.30550 & 2.30575 \\
4.2 & 2.49806 & 2.49720 & 2.49805 \\
4.4 & 2.69238 & 2.68965 & 2.69242 \\
4.6 & 2.88826 & 2.88002 & 2.88859 \\
4.8 & 3.08534 & 3.06157 & 3.08718 \\
5 & 3.28239 & 3.21785 & 3.29272 \\
\hline
\end{tabular}

To complete the solution, we choose $f_{0}(\eta)=F(0)=f(0)=0, F^{\prime}(0)=f^{\prime}(0)=0$, and $F^{\prime \prime}(0)=f^{\prime \prime}(0)=\sigma$. Solving $(4.8)$ for $F_{j}(\eta), j=0,1, \ldots$, leads to the results

$$
\begin{aligned}
F_{0}(\eta) & =\frac{1}{2} \sigma x^{2} \\
F_{1}(\eta) & =-\frac{1}{240} \sigma^{2} x^{5} \\
F_{2}(\eta) & =\frac{11}{161280} \sigma^{3} x^{8} \\
F_{3}(\eta) & =-\frac{5}{4257792} \sigma^{4} x^{11} \\
F_{4}(\eta) & =\frac{9299}{464950886400} \sigma^{5} x^{14}, \\
F_{5}(\eta) & =-\frac{127239}{3793999233024000} \sigma^{6} x^{17}, \\
F_{6}(\eta) & =\frac{19241647}{3460127300517888000} \sigma^{7} x^{20} \\
\vdots &
\end{aligned}
$$


Table 2: Comparison between the Howarth and LTNHPM methods for $f^{\prime}(\eta)$.

\begin{tabular}{|c|c|c|c|}
\hline$\eta$ & Howarth [2] & $\mathrm{g}^{*}(\eta)$ & $\mathrm{g}^{* *}(\eta)$ \\
\hline 0 & 0.00000 & 0.00000 & 0.00000 \\
\hline 0.2 & 0.06641 & 0.06641 & 0.06641 \\
\hline 0.4 & 0.13277 & 0.13276 & 0.13276 \\
\hline 0.6 & 0.19894 & 0.19894 & 0.19894 \\
\hline 0.8 & 0.26471 & 0.26471 & 0.26471 \\
\hline 1 & 0.32979 & 0.32978 & 0.32978 \\
\hline 1.2 & 0.39378 & 0.39378 & 0.39378 \\
\hline 1.4 & 0.45627 & 0.45626 & 0.45626 \\
\hline 1.6 & 0.51676 & 0.51676 & 0.51676 \\
\hline 1.8 & 0.57477 & 0.57476 & 0.57476 \\
\hline 2 & 0.62977 & 0.62977 & 0.62977 \\
\hline 2.2 & 0.68132 & 0.68131 & 0.68131 \\
\hline 2.4 & 0.72899 & 0.72898 & 0.72898 \\
\hline 2.6 & 0.77246 & 0.77245 & 0.77245 \\
\hline 2.8 & 0.81152 & 0.81151 & 0.81151 \\
\hline 3 & 0.84605 & 0.84604 & 0.84604 \\
\hline 3.2 & 0.87609 & 0.87607 & 0.87608 \\
\hline 3.4 & 0.90177 & 0.90173 & 0.90176 \\
\hline 3.6 & 0.92333 & 0.92321 & 0.92333 \\
\hline 3.8 & 0.94112 & 0.94067 & 0.94112 \\
\hline 4 & 0.95552 & 0.95396 & 0.95553 \\
\hline 4.2 & 0.96696 & 0.96192 & 0.96704 \\
\hline 4.4 & 0.97587 & 0.96047 & 0.97639 \\
\hline 4.6 & 0.98269 & 0.93806 & 0.98564 \\
\hline 4.8 & 0.98779 & 0.86475 & 1.00322 \\
\hline 5 & 0.99155 & 0.66723 & 1.06671 \\
\hline
\end{tabular}

Therefore, we gain the solution of (2.8) as

$$
\begin{aligned}
f(\eta) & =\lim _{p \rightarrow 1} \sum_{n=0}^{\infty} p^{n} F_{n}(\eta) \\
& =F_{0}(\eta)+F_{1}(\eta)+F_{2}(\eta)+\cdots \\
& =\frac{1}{2} \sigma x^{2}-\frac{1}{240} \sigma^{2} x^{5}+\frac{11}{161280} \sigma^{3} x^{8}-\frac{5}{4257792} \sigma^{4} x^{11}+\cdots
\end{aligned}
$$

According to Howarth calculation [2], inserting $\sigma=0.332057$, therefore, the analytical approximation to the solution of Blasius equation can be expressed as

$$
\begin{aligned}
f(\eta)= & 0.1660285000 \eta^{2}-0.0004594243800 \eta^{5}+0.000002497181392 \eta^{8} \\
& -1.427697248 \times 10^{-8} \eta^{11}+8.074067341 \times 10^{-11} \eta^{14}-4.495676921 \times 10^{-13} \eta^{17}+\cdots
\end{aligned}
$$


Suppose $f^{*}(\eta)=\sum_{n=0}^{12} F_{n}(\eta), f^{* *}(\eta)=\sum_{n=0}^{7} F_{n}(\eta), g^{*}(\eta)=(d / d \eta) f^{*}(\eta)$, and $g^{* *}(\eta)=$ $(d / d \eta) f^{* *}(\eta)$, some numerical results of these solutions are presented in Tables 1 and 2, Figures 1 and 2.

Table 1 is made to compare between present results and results given by Howarth [2]. In Figures 1 and 2, one can also see the comparison between LTNHPM results and Howarth's results.

\section{Conclusion}

In this paper, the combined Laplace transform and homotopy perturbation methods are employed to give numerical solutions of the classical Blasius flat-plate flow in fluid mechanics. To illustrate the accuracy and efficiency of the proposed procedure, various different examples in the interval $0 \leq \eta \leq 5$ have also been analyzed and the numerical results are listed in Tables 1 and 2. Also, we have compared, in Figures 1 and 2, the numerical values of $f$ and $f^{\prime}$ with those of Howarth [2]. The results are found to be in good agreement. The results show that the LTNHPM is an effective mathematical tool which can play a very important role in nonlinear sciences.

\section{References}

[1] H. Blasius, "The boundary layers in fluid with little friction," Zeitschrift fur Mathematik und Physik, vol. 56, no. 1, 908, pp. 1-37, 1950 (German), English translation available as NACATM 1256.

[2] L. Howarth, "On the solution of the laminar boundary-layer equations," Proceedings of the Royal Society of London, vol. 164, pp. 547-579, 1983.

[3] A. M. M. Abu-Sitta, "A note on a certain boundary-layer equation," Applied Mathematics and Computation, vol. 64, no. 1, pp. 73-77, 1994.

[4] A. Asaithambi, "A second-order finite-difference method for the Falkner-Skan equation," Applied Mathematics and Computation, vol. 156, no. 3, pp. 779-786, 2004.

[5] J. He, "Approximate analytical solution of blasius' equation," Communications in Nonlinear Science and Numerical Simulation, vol. 4, no. 1, pp. 75-78, 1999.

[6] S. Abbasbandy, "A numerical solution of Blasius equation by Adomian's decomposition method and comparison with homotopy perturbation method," Chaos, Solitons and Fractals, vol. 31, no. 1, pp. 257$260,2007$.

[7] M. Esmaeilpour and D. D. Ganji, “Application of He's homotopy perturbation method to boundary layer flow and convection heat transfer over a flat plate," Physics Letters A, vol. 372, no. 1, pp. 33-38, 2007.

[8] H. Aminikhah and M. Hemmatnezhad, "An efficient method for quadratic Riccati differential equation," Communications in Nonlinear Science and Numerical Simulation, vol. 15, no. 4, pp. 835-839, 2010. 


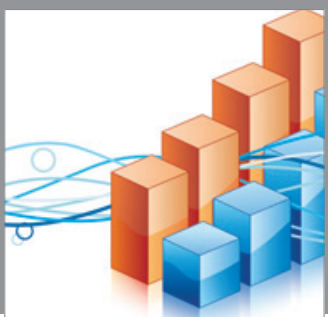

Advances in

Operations Research

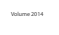

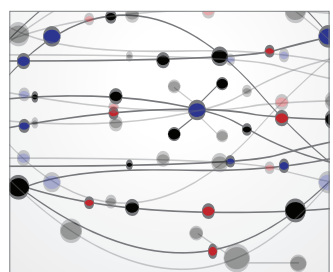

\section{The Scientific} World Journal
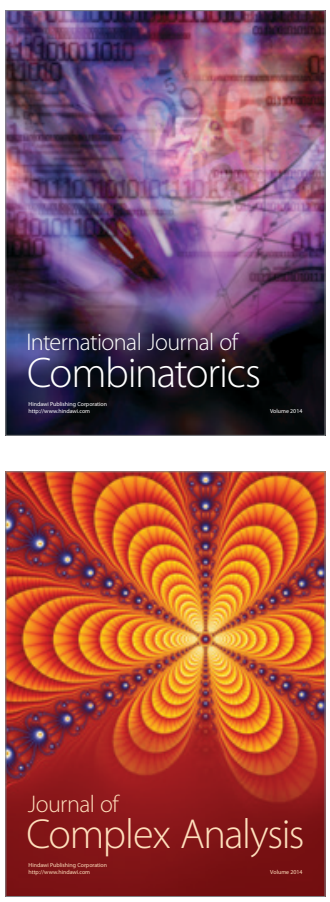

International Journal of

Mathematics and

Mathematical

Sciences
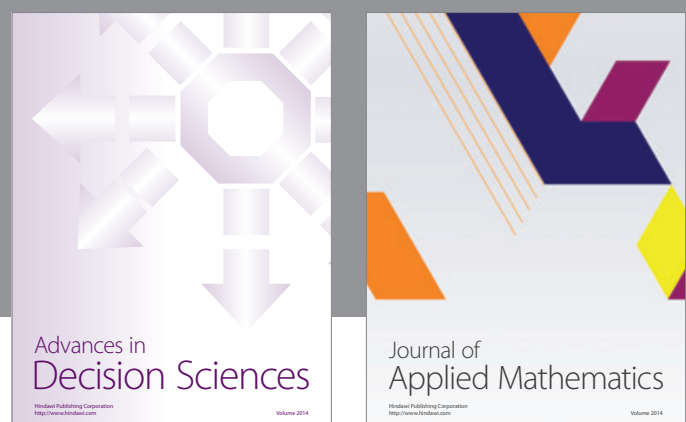

Journal of

Applied Mathematics
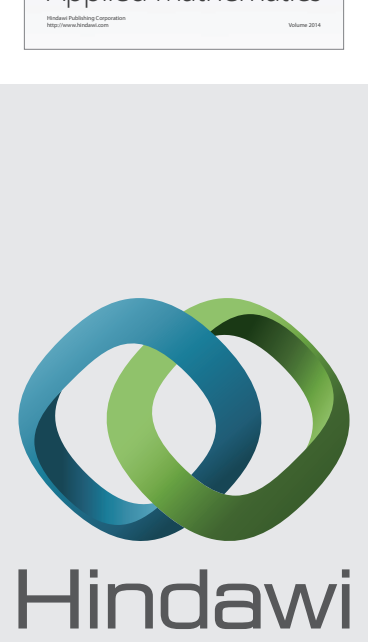

Submit your manuscripts at http://www.hindawi.com
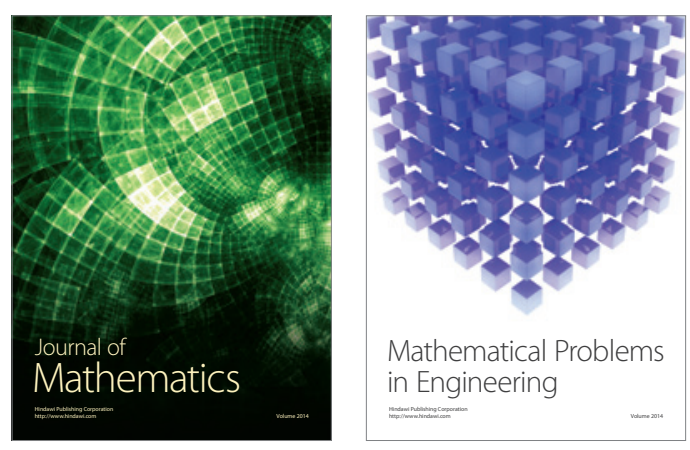

Mathematical Problems in Engineering
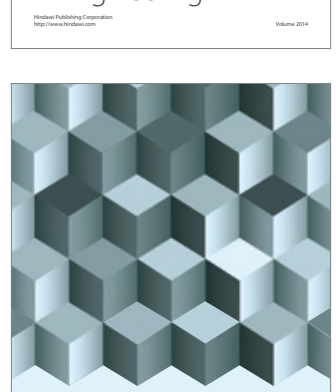

Journal of

Function Spaces
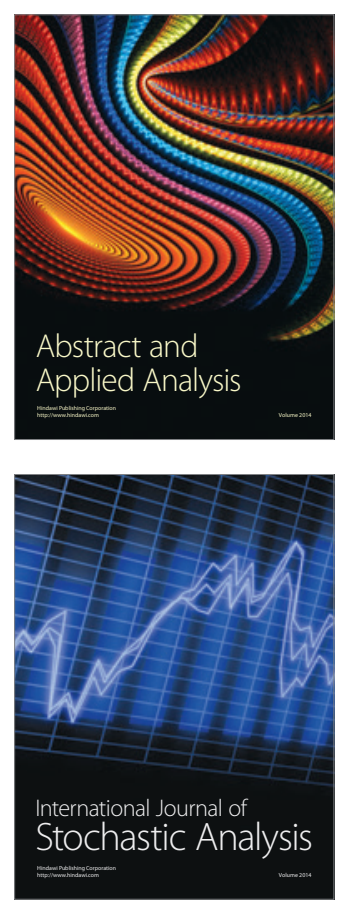

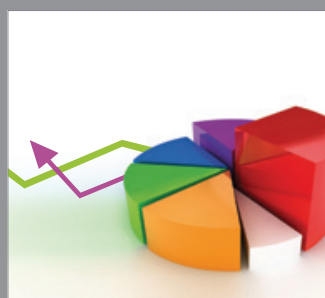

ournal of

Probability and Statistics

Promensencen
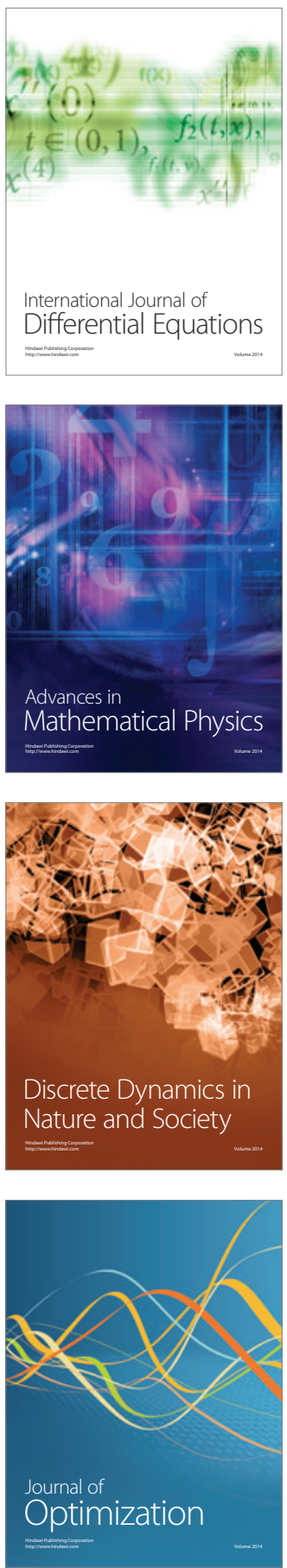